PPPL-3460

PPPL-3460

UC-70

Rotation and Particle Loss in Tore Supra

by

R. B. White, F. W. Perkins, X. Garbet, C. Bourdelle,

V. Basiuk, and L. G. Eriksson

June 2000

$\int D \sqrt{S} \sqrt{5} \iint \begin{aligned} & \text { PAINCETON } \\ & \text { PLABMA PHYSICS } \\ & \text { LABOAATOAY }\end{aligned}$

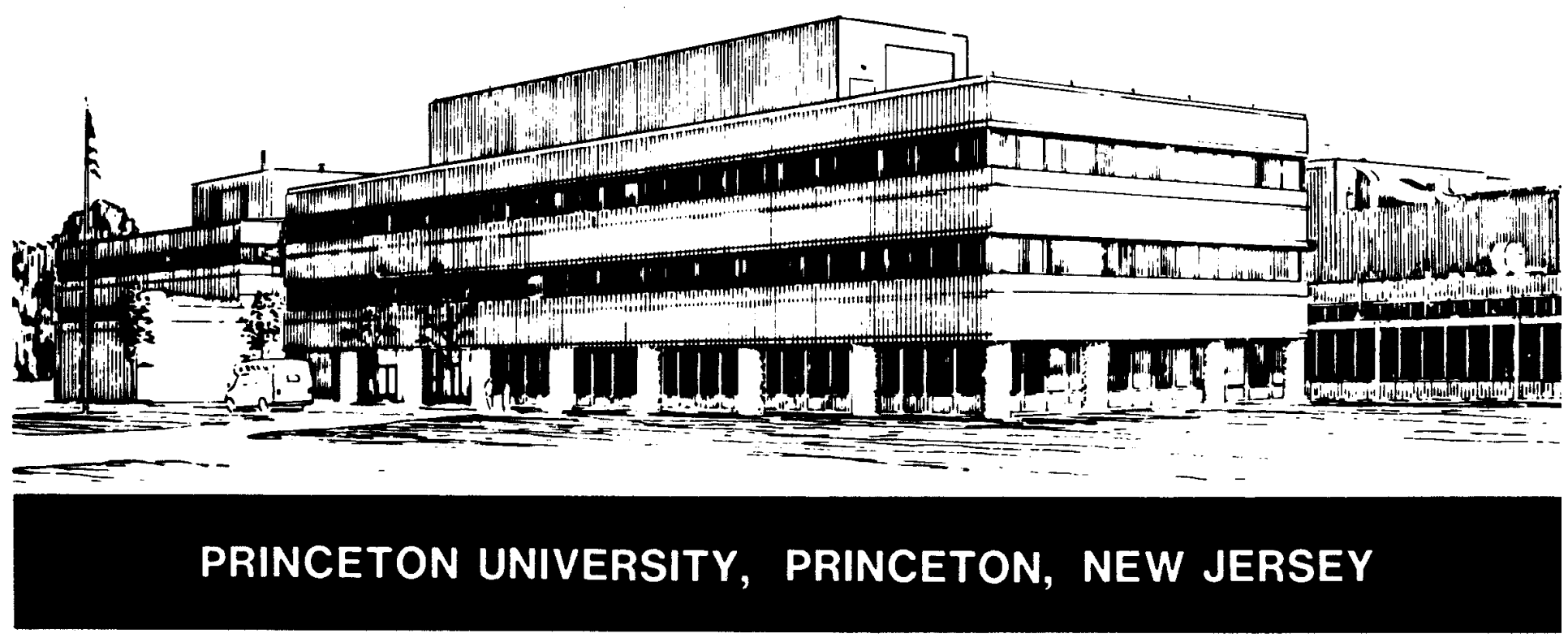




\section{PPPL Reports Disclaimer}

This report was prepared as an account of work sponsored by an agency of the United States Government. Neither the United States Government nor any agency thereof, nor any of their employees, makes any warranty, express or implied, or assumes any legal liability or responsibility for the accuracy, completeness, or usefulness of any information, apparatus, product, or process disclosed, or represents that its use would not infringe privately owned rights. Reference herein to any specific commercial product, process, or service by trade name, trademark, manufacturer, or otherwise, does not necessarily constitute or imply its endorsement, recommendation, or favoring by the United States Government or any agency thereof. The views and opinions of authors expressed herein do not necessarily state or reflect those of the United States Government or any agency thereof.

\section{Availability}

This report is posted on the U.S. Department of Energy's Princeton Plasma Physics Laboratory Publications and Reports web site in Calendar Year 2000. The home page for PPPL Reports and Publications is: http://www.pppl.gov/pub_report/

DOE and DOE Contractors can obtain copies of this report from:

U.S. Department of Energy

Office of Scientific and Technical Information

DOE Technical Information Services (DTIS)

P.O. Box 62

Oak Ridge, TN 37831

Telephone: (865) 576-8401

Fax: (865) 576-5728

Email: reports@adonis.osti.gov

This report is available to the general public from:

National Technical Information Service

U.S. Department of Commerce

5285 Port Royal Road

Springfield, VA 22161

Telephone: $1-800-553-6847$ or

(703) $605-6000$

Fax: (703) 321-8547

Internet: http://www.ntis.gov/ordering.htm 


\title{
Rotation and Particle Loss in Tore Supra
}

\author{
R. B. White ${ }^{1}$, F. W. Perkins ${ }^{2}$, X. Garbet ${ }^{3}$, C. Bourdelle ${ }^{3}$, V. Basiuk ${ }^{3}$, L. \\ G. Eriksson ${ }^{3}$ \\ ${ }^{1}$ Plasma Physics Laboratory, Princeton University, P.O.Box 451, \\ Princeton, New Jersey 08543, ${ }^{2}$ General Atomics, San Diego, Ca, \\ ${ }^{3}$ Assoc. CEA Euratom, Cadarache, France
}

Although plasma heating with ICRF imparts negligible angular momentum to a tokamak plasma, the high energy particles give significant torque to the plasma through diamagnetic effects. This effect has been directly modeled through guiding center simulations. It is found that heating in Tore Supra, with the location of the resonance surface on the high field side of the magnetic axis, can produce negative central rotation of up to $40 \mathrm{~km} / \mathrm{sec}[1,2]$. Particle loss also contributes to negative rotation, but this is not the dominant effect in most discharges. In this work we examine the effect of collisions and strong plasma rotation on the loss of high energy particles.

Magnetic field strength variation due to discrete toroidal field coils, or ripple, produces two important loss channels in tokamaks. The trapping of particles in local ripple wells produces super banana orbits and, in the case of strong ripple, direct loss orbits leading to the plasma edge. These particles leave the device in the direction of vertical drift, and are characterized by small values of parallel velocity, or pitch. Ripple also causes high energy particles in banana orbits to diffuse stochastically [3, 4], leading to banana orbits which impact the wall near the outer midplane. Both these loss processes are modified by the magnitude of the collision rate, and by plasma rotation. In Tore Supra the magnitude of the ripple makes ripple trapping a dominant loss mechanism for the background plasma as well as for ICRF produced non Maxwellian high energy tails. We have examined the loss as a function of collisionality and rotation using the Hamiltonian guiding center code ORBIT $[5,6]$.

Although the simulations make use of a numerical equilibrium with a Shafranov shift closely matching the experiments, we use simple circular equilibrium expressions in the discussion of analytic estimates. Represent the field as $B \sim B_{0}(r, \theta)(1+\delta \sin N \phi)$ with $\delta$ giving the ripple strength, $\mathrm{r}$ the minor radius, and $\theta$ the poloidal and $\phi$ the toriodal angles. Along a field line $\phi=q \theta, r=$ constant so a ripple well boundary is given by $\partial_{\theta} B_{0}+N q \delta B_{0}=0$. In simple circular geometry with $B_{0} \sim 1-r \cos \theta$ wells exist if $r|\sin \theta|<N q \delta$, a condition which is independent of particle energy. To remain in a ripple well a particle must have pitch $\lambda=v_{\|} / v$ less than $\sqrt{\delta}$. The ripple well domain and the stochastic loss domain in Tore Supra for the equilibrium studied are shown in Fig. 1. In the figure to the left the shaded domain shows the extent of the ripple wells. A ripple trapped particle drifts downward at a rate given by $v_{d}=(\rho / R)^{2} R \omega_{0}$ with $\rho$ the gyro radius, $\omega_{0}$ the gyro frequency and $R$ the major radius. Once a particle is ripple trapped in a loss orbit leading to the wall the loss process typically takes a fraction of a millisecond. Particles which are ripple trapped drift downward a distance $\Delta z$ until they leave the ripple well, either collisionlessly or due to a collision, with the maximum value of $\Delta z$ determined by the geometry of the domain shown in Fig. 1. Particles can alternate between ripple well traps and banana orbits with each trapping in a well producing an outward shift[7] by a distance $z \Delta z / r$, (the superbanana width) leading ultimately to an 

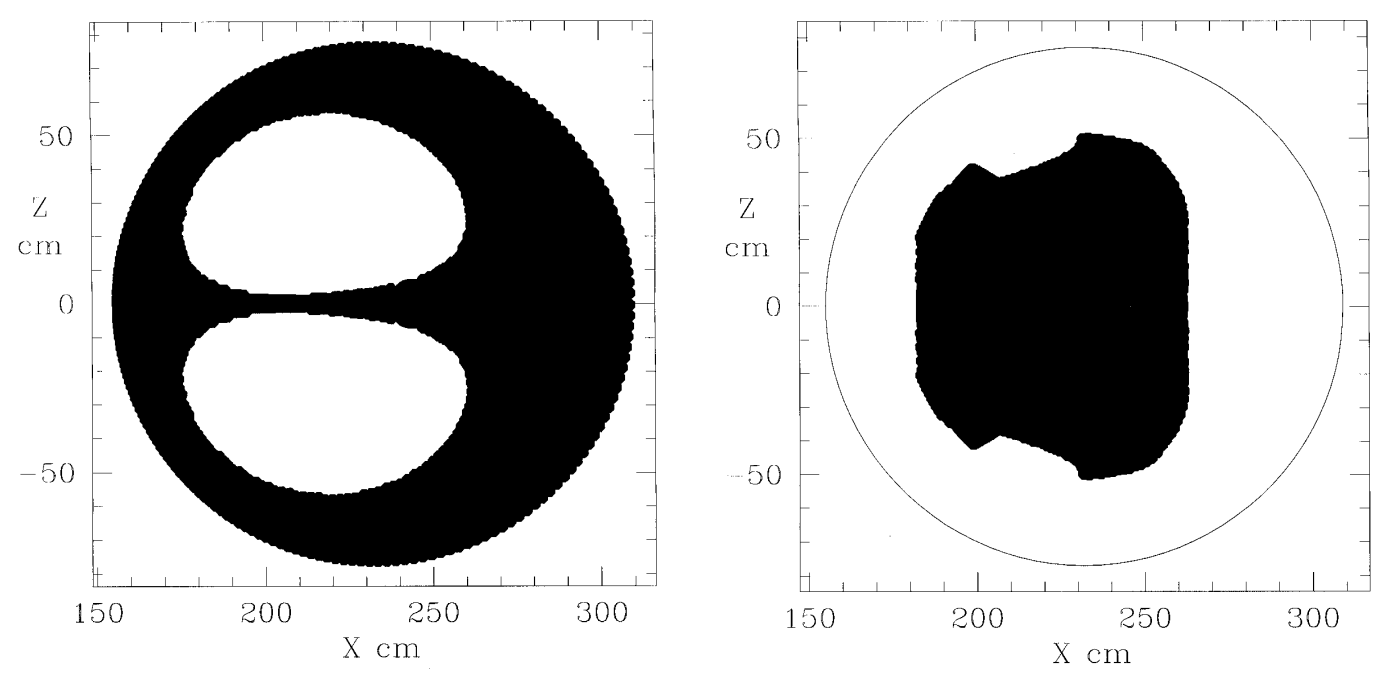

Figure 1: Ripple wells (left,shaded), and stochastic loss domain for $100 \mathrm{keV}$ ions (right, not shaded)

orbit which reaches the wall. The figure to the right shows the stochastic loss domain. All particles with bounce point outside the shaded area are lost diffusively. Although this domain is also extensive, we will see that ripple trapping is the dominant ultimate loss mechanism.

Pitch angle scattering with a collision frequency $\nu$ modifies the loss by scattering particles in and out of ripple trapped orbits, and in making both the ripple trap domain and the stochastic loss domain ultimately accessible to all particles. If the effective collision frequency $\nu_{\text {eff }}$ is less than the reciprical of the loss time $1 / T_{\text {loss }}$ the steady state loss is limited by the scattering of particles into the loss domain, and is thus proportional to $\nu$. For $\nu$ larger than this the loss process scales with $1 / \nu$, since the effect of the collisions is to depopulate the loss domain.

We have analyzed losses in shot 23418 for both the main plasma and for the ICRF heated tail. The toroidal field strength was 3.7 Tesla and the magnetic axis was at $244 \mathrm{~cm}$ with a $12 \mathrm{~cm}$ Shafranov shift. Central ion temperature was $3.25 \mathrm{keV}$. A simple pitch angle scattering operator was used to investigate the effects of collisions[8]. It is easy to distinguish the ripple trapped loss from the banana orbit loss by the fact that all loss with small $|\lambda|$ is due to ripple trapping. The two loss processes for the background plasma as a function of collision frequency are shown in Fig. 2. Losses in Tore Supra are dominated by ripple trapping for normal values of collisionality. This however does not mean that stochastic diffusion is unimportant, only that the ultimate loss process is normally ripple trapping. It is possible for a particle to experience extensive stochastic ripple diffusion before finally being trapped in a ripple well. If the pitch distribution of the ripple trapped particles is $\delta \lambda$ and the loss time is $\delta t$ a collision frequency much larger than $(\delta \lambda)^{2} / \delta t$, should detrap particles before they are lost. This is seen in Fig. 2 in the appearance of an approximate $1 / \nu$ dependence of the ripple trapped loss for large collisionality. Simulations give $\delta \lambda \sim 0.3$ for ripple trapping, giving the transition point at $\nu \sim 200 /$ sec.

For purposes of the simulations only ICRF particles with energy above $50 \mathrm{keV}$ were kept, so that only the high energy tail was simulated. The distribution in pitch at the midplane 

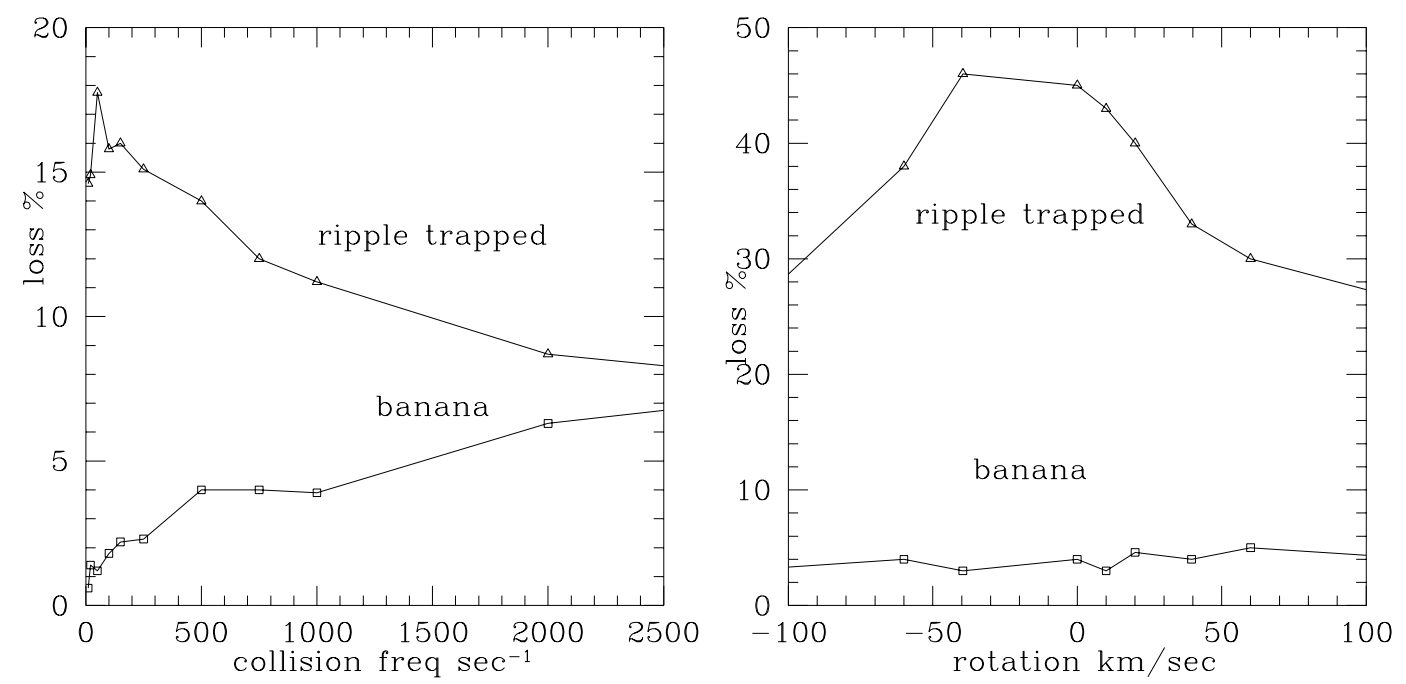

Figure 2: Thermal particle loss vs collisions (left) and ICRF loss vs rotation (right)

on the low field side was given by a rabbit ears distribution

$$
f=e^{-\left(\lambda-\lambda_{0}\right)^{2} / \sigma^{2}}+e^{-\left(\lambda+\lambda_{0}\right)^{2} / \sigma^{2}}
$$

with the width $\sigma \simeq 25 / E_{k e v}$, and $\lambda=v_{\|} / v$ the pitch, with $\lambda_{0}$ that value of the pitch resulting in the bounce point being located at the resonance layer, $X=234 \mathrm{~cm}$, i.e. located $10 \mathrm{~cm}$ on the high field side of the magnetic axis. Approximately $3 / 4$ of the particles above $50 \mathrm{keV}$ are trapped, due to the localization of high energy particles with bounce points near the resonance layer.

Plasma rotation effects become important, provided the plasma is in a relatively collisionless domain, when the rotation velocity is comparable to the drift velocity. Rotation of the trapped particle population due to a radial electric field, and precession of a deeply trapped particle are given by

$$
\frac{\omega_{\phi}}{\omega_{0}}=-\frac{q}{r} \frac{\partial_{r} \Phi}{2 E} \rho^{2}, \quad \frac{\omega_{d}}{\omega_{0}}=\frac{q \rho^{2}}{2 r R} .
$$

with $\Phi$ the electric potential energy, $\omega_{\phi}$ the toroidal rotation rate, $\omega_{d}$ the toroidal precession, $\omega_{0}$ the gyro frequency, $\rho$ the gyro radius, and $\mathrm{E}$ the particle energy. One effect of this potential on the ripple trapped particles is to change the drift direction. Energy conservation for a ripple trapped particle, since the parallel velocity is very small, is $\Phi(R, z)+\mu B(R)=E$. While the particle is trapped, the bounce motion in the well adjusts the toroidal location so as to keep the particle near the ripple minimum. The orbit path in $\mathrm{R}$ and $\mathrm{z}$ is thus given by

$$
\frac{d R}{d z} \simeq \frac{-\partial_{z} \Phi}{\mu \partial_{R} B}
$$

where we have assumed that $\mu \partial_{R} B \gg \partial_{R} \Phi$. For positive rotation we have $\partial_{r} \Phi<0$ and thus below the midplane $\partial_{z} \Phi>0$ which means that the orbit is curved toward smaller $\mathrm{R}$ as it drifts downward. This makes the superbanana width smaller, and also causes the particle to leave the ripple well trap earlier. This effect becomes important when 
$r \partial_{r} \Phi \sim(r / R) E$ giving $\omega_{\phi} / \omega_{0} \simeq \rho^{2} /(R r)$. The effect of this shrinking of the superbanana width is to decrease the loss rate.[7] The superbanana width becomes roughly $\delta r=\left(v_{d} z \Delta z / r\right) /\left(v_{E}+v_{d}\right)$ where $v_{d}$ is the vertical drift velocity and $v_{E}$ is the rotation velocity. The rotation decreases transport only in the low collision domain, where transport is dominated by ripple trapped particles.

Negative rotation instead increases the size of the superbanana and moves the particle towards larger ripple. Since the downward drift, proportional to the energy, is in competition with the collisional detrapping, this should increase the loss by allowing more low energy particles to participate in the trapped loss[7]. However for negative rotation and $r \partial_{r} \Phi \sim E$ the potential prevents the loss of particles simply due to energy conservation. This gives $\omega_{\phi} / \omega_{0} \simeq \rho^{2} / r^{2}$, giving for a particle of $E=1 \mathrm{keV}$ a frequency of $\omega_{\phi} \simeq 300 / \mathrm{sec}$. Although beginning at somewhat larger frequencies, this effect is stronger than orbit modification effects, and furthermore is independent of whether the particle is ripple trapped, and thus negative rotation inhibits ion loss.

The stochastic loss domain is also modified by rotation. Increased rotation is, in terms of the orbital dynamics, equivalant to increased energy since the toroidal drift rate determines the resonance phenomena[4]. Positive rotation thus acts to increase the size of the stochastic ripple loss domain, and negative rotation to decrease it.

As seen in Fig. 2, with the collision rate held at 500/sec, the simulations for the ICRF tail show that rotation gives a decrease in the ripple trapped loss, only slightly compensated by an increase in banana particle loss. The observed rotation rate was $-40 \mathrm{~km} / \mathrm{sec}$ or $\omega_{\phi}=-1.6 \times 10^{4} / \mathrm{sec}$.

To conclude, we find that in Tore Supra particle loss to the edge is dominated by ripple trapping, both for the bulk plasma and for the heated ICRF high energy tail, both with and without observed rotation rates. Stochastic ripple diffusion, although present, normally ends in a ripple trapped loss trajectory before bringing a particle into contact with the wall. Plasma rotation of either sign acts to diminish the ripple trapped loss, but the effect is not important except for very fast rotation. This effect was predicted by Yushmanov[7] for positive rotation, the decrease in the ripple loss for negative rotation is due to energy conservation. For the ICRF high energy tail particles plasma rotation increases the stochastic ripple loss domain. For both particle distributions positive rotation tends to a transition from ripple trapped loss to banana orbit loss, although the effect is small for energetic ions. Negative rotation decreases both types of loss, simply through the potential barrier prohibiting motion to the edge.

This work was supported by the U.S. Department of Energy under contract number DE-AC02-76CHO3073, and by the Universite de Provence, Marseilles, where one of the authors (RBW) was a visiting Professor.

[1] Eriksson, L.-G. et. al., Plasma Phys. Control. Fusion 39, 27 (1997).

[2] F. W. Perkins, R. B. White, EPS, this meeting .

[3] R. J. Goldston, R. B. White and A. H. Boozer, Phys Rev Lett 47, 647 (1981).

[4] R. B. White, Phys Rev E 58, 1774 (1998).

[5] R. B. White, and M. S. Chance, Phys Fluids 27, 2455 (1984).

[6] R. B. White, Phys Fluids B 2, 845 (1990).

[7] P. N. Yushmanov, Nucl. Fus. 22, 315 (1982).

[8] A. H. Boozer and G. Kuo-Petravic, Physics Fluids 24, 851 (1981). 
The Princeton Plasma Physics Laboratory is operated by Princeton University under contract with the U.S. Department of Energy.

\author{
Information Services \\ Princeton Plasma Physics Laboratory \\ P.O. Box 451 \\ Princeton, NJ 08543
}

Phone: 609-243-2750

Fax: 609-243-2751

e-mail: pppl_info@pppl.gov

Internet Address: http://www.pppl.gov 